\title{
„..Da kenn ich eine kleine Kirche.. da gehe ich rein.. bete ich tïrkisch...". Modifikationen religiöser Identitäten bei Akademikerinnen türkischer Herkunft in Wien
}

Sule Dursun

The following paper aims to present religious identities of young, educated Turkish women in Vienna. Analysis of the interviews shows that university education leads to individual modification of religious and cultural practices as well as an adaptation of religious values and cultural attitudes in the lives of these women. The results show that young women - who were brought up in a conservative or popular Islamic way - developed a modified, individual understanding of their religion. The approach of religious reflexivity as a result of continuous confrontation with new experiences is the decisive result of the study. These young women reflected upon their upbringing/setting and relied on themselves and their own intuition to solve religious challenges.

Sule Dursun earned her doctoral degree in Islamic Religious Education at the University of Vienna. Besides her activity as Islamic teacher of religion at public and vocational schools she teaches kindergarten pedagogues in the subject of Islamic religion. Her research interests are: Islamic religious education, interreligious dialogue and religious beliefs of Muslims with Turkish provenance.

\section{Einführung}

Befasst man sich mit den aktuellen öffentlichen Diskursen bzw. Debatten über „die“ muslimische Frau im europäischen Kontext, so fällt auf, dass zumeist von einem problemzentrierten Fokus ausgegangen wird.

Die Debatten über die muslimische Frau arbeiten sich vorwiegend an deren Sichtbarkeit in der Öffentlichkeit ab und da vor allem an einem zur europäischen Kultur als widersprüchlich wahrgenommenen Bekleidungsstil.

So löste beispielweise das Gesetz über das Gesichtsschleierverbot in Frankreich aus dem Jahr 2010, das nicht explizit an die muslimische Frau adressiert war, aber dennoch vor allem für muslimische Frauen mit Burka (Vollverschleierung) eine strafrechtliche Verfolgung mit sich brachte, ${ }^{1}$ noch vor Jahresende 2015 auch in Deutschland eine Diskussion aus, ob dieses Verbot per Gesetzeserlass einge-

1 Vgl. Internetquellen: Hölzl. 
führt werden solle. ${ }^{2}$ Ein Jahr früher als in Deutschland wurde in Österreich 2014 die gleiche Debatte geführt und ein Gesetzeserlass zum Burkaverbot im Parlament abgelehnt. ${ }^{3}$

$\mathrm{Zu}$ Hauptfiguren in den Berichterstattungen werden häufig muslimische Frauen, die viele Jahre ein Kopftuch getragen hatten, das Ablegen des Kopftuches nunmehr als eine Befreiung wahrnehmen und für eine kritische Betrachtungsweise des Kopftuches plädieren. ${ }^{4}$ Zugleich wird in der öffentlichen Darstellung im EU-Raum meist eine Dichotomie aufgespannt, wie etwa jene, dass das Ablegen des Kopftuches in Saudi Arabien mit Hinrichtung enden könnte. Dieser transnationale Vergleich bringt die gängige Stereotypisierung des Islam mit sich, dass der Islam generell demokratiefeindlich sei oder die muslimische Frau zur Wahrung streng traditioneller Werte verpflichtet werde. ${ }^{5}$

In diesen vorgefertigten Klischees scheint die Emanzipation ${ }^{6}$ der muslimischen Frau tendenziell nur dann für möglich gehalten zu werden, wenn sie sich von ihrer eigenen Religion und Kultur distanziert.

Quasi als „Illustration“ dieser klischeebehafteten Annäherungen an die Thematik zeigt sich aber tatsächlich auch ein traditionelles bzw. religiös geprägtes Frauenbild in vielen türkischen Familien in Europa, das als ein Widerspruch zu den Emanzipationsvorstellungen der Postmoderne gedeutet werden kann. In diesen Familien beobachtet man eher ein religiös traditionelles Frauenbild, in dem der Platz der Frau in erster Linie auf ihr Haus und ihre Familie beschränkt ist.

Der Fokus des vorliegenden Artikels auf gebildete Frauen ergab sich u. a. daraus, dass die Bildung der von Musliminnen als Zauberformel für deren Emanzipation und Integration betrachtet wird. Beispielsweise wurde im Juni 2012 eine Entscheidung des Europarates getroffen, aufgrund welcher muslimische Frauen, die in den Medien zumeist als „Opfer“ oder „unterdrückte Frauen im Islam" dargestellt werden, nun seitens der EU-Staaten zu mehr Bildung ermutigt werden, und durch die ihnen eine chancengerechte Teilnahme an Arbeit und Bildung ohne Stereotypisierung ermöglicht werden soll. ${ }^{7}$

Der vorliegende Beitrag diskutiert den Einfluss der höheren Bildung auf die religiösen Einstellungen muslimischer Frauen türkischer Herkunft.

Im zweiten Kapitel werden Studien zur Religiosität von MuslimInnen unter die Lupe genommen, wobei jeweils festgestellt werden kann, dass auch diese auf einen religiösen Wandel im Leben der MuslimInnen mit höherer Bildung hinweisen. Vermisst wird in diesen Studien ein umfassender Bezug zu Akademikerinnen türkischer Herkunft aus Wien und die Rolle der Religion in ihrem Leben,

2 Vgl. Internetquellen: OE24.

3 Vgl. Internetquellen: News.

4 Vgl. Internetquellen: Zeynelabidin.

5 Vgl. ebd.

6 Vgl. Mader 2009, S. 27.

7 Vgl. Internetquellen: Kyriakidou. 
unabhängig davon, wie intensiv sie religiöse Praxis in ihrem Leben berücksichtigen.

Kapitel drei widmet sich dem handbuchorientieren Islamverständnis der MuslimInnen türkischer Herkunft, da dieser Mainstream sich bei den Interviewpartnerinnen als ursprünglicher Bezugspunkt herausstellte.

Anhand exemplarischer Stellungnahmen vieler muslimischer Interviewpartnerinnen zeigt der folgende Beitrag, dass sich die religiöse Identität der jungen Frauen türkischer Herkunft durch ihren akademischen Werdegang auf eine beachtliche Art und Weise verändert hat. Sofern die Frauen vor ihrem Bildungsprozess bzw. ihrer Erwerbstätigkeit von einem konservativen oder volkstümlichen Islam ausgegangen sind, charakterisiert sich die Transformation ihrer religiösen Identität vor allem als eine individuellere Modifikation ihrer religiösen und kulturellen Praxis. Diese Veränderung hat sich bei den einen eher intuitiv, bei den anderen infolge einer explizit reflexiven Auseinandersetzung vollzogen. Die reflexive Religiosität der Interviewpartnerinnen stärkt diese auch in ihren Emanzipationsidealen, in ihrer gesellschaftlichen Positionierung und in ihrem Familienleben. ${ }^{8}$

\section{Studien zur Religiosität von Muslimen mit höherer Bildung}

Forschungsarbeiten, die sich speziell mit der Religiosität von Muslimen mit höherer Bildung befassen, sind selten. Die folgenden Forschungsarbeiten zeigen einen religiösen Wandel der Muslime türkischer Herkunft. Ausgehend von den überblicksmäßig ausgeführten Forschungsarbeiten fokussiert dieser Beitrag die unterschiedlichen Erzählungen meiner Interviewpartnerinnen über Religion.

In ihrer Dissertation „Religiöse Orientierungen und Erziehungsvorstellungen“, befragt Yasemin Karakaşoğlu im Rahmen einer qualitativ-empirischen Untersuchung angehende Pädagoginnen türkisch-muslimischer Herkunft in Deutschland. ${ }^{9}$ Mit Hilfe des Religionspsychologen Glock entwickelten Dimensionen der Religiosität analysiert Karakaşoğlu die verschiedenen Dimensionen der Religiosität der Frauen und identifiziert sechs Typen religiöser Orientierung: Atheistinnen, Spiritualistinnen, sunnitische Laizistinnen, pragmatische Ritualistinnen und idealistische Ritualistinnen. Ihre Untersuchung ergibt u.a., dass die Religiosität im Leben der sunnitischen und alevitischen Musliminnen eine ethische Grundorientierung, eine Quelle mentaler Kraft und einen wichtigen kulturellen Bezugspunkt darstellt. Ebenso wird die von Eltern tradierte traditionelle Religiosität als falsches und unzeitgemäßes Verständnis empfunden. Die Befragten

8 Vgl. Dursun 2015.

9 Vgl. Karakaşoğlu 2003, S. 272 f. 
betonten hierbei ihren intellektuellen Zugang und die Bedeutung der Bildung für ihre religiöse Orientierung. ${ }^{10}$

Ein weiteres aufschlussreiches Projekt zu Religion und Höherer Bildung wurde von der niederländischen Theologin Manuela Kalsky durchgeführt. Sie analysiert in ihrem Buch „Modern Devoties“ die Religiosität von Frauen unterschiedlicher Religionszugehörigkeiten aus den Niederlanden, die aus heterogenen Berufsfeldern stammten (aus Politik, Literatur und anderen Bereiche des öffentlichen Lebens in den Niederlanden). Sie geht der Frage nach, wie diese emanzipierten Frauen heute glauben, welche Aspekte ihrer jeweiligen Glaubenstradition sie in ihrem persönlichen und gesellschaftlichen Leben als inspirierend und wegweisend erfahren haben, und welche Rolle ihr Glaube im täglichen Leben spielt. Kalsky kam zu dem Ergebnis, dass diese Frauen kritisch, selbstbewusst und transformativ mit ihren religiösen Traditionen umgehen und lediglich jene Glaubensinhalte tradieren, die in ihrer konkreten Lebens- und Glaubenspraxis von Bedeutung sind. ${ }^{11}$

Eine weitere relevante Studie, die sich auf gebildete Frauen bezieht, ist die Forschungsarbeit von Brigitte Jelen, Dozentin an der Bahçeşehir Universität in Istanbul aus dem Jahr 2011. In ihrer Studie „Educated, Independent, and Covered: the Professional Aspirations and Experiences of University-Educated Hijabi in Contemporary Turkey" kommt sie zu dem Ergebnis, dass diese jungen Frauen obwohl sie eine Minderheit in Istanbul darstellen - trotz diverser Schwierigkeiten versuchen, ihre modernen bzw. unabhängigen Lebensstile mit der islamischen orthodoxen Lebensweise zu vereinbaren. Jelen stellt fest, dass viele dieser hochqualifizierten Frauen alleine leben, gerne reisen, Gitarre spielen bzw. einige von ihnen erfolgreiche Autorinnen sind. Ebenso betont Jelen in ihren Studienergebnissen, dass sie sich von Alkohol distanzieren, regelmäßig beten und sich von vorehelichen geschlechtlichen Beziehungen fernhalten. ${ }^{12}$ Die Forschungsergebnisse von Jelen zeigen eine Zusammenführung des modernen und orthodoxen Lebensstils. Die Studie erfasst allerdings nicht die Einstellungen von gebildeten Frauen, die kein Kopftuch tragen.

Eine repräsentative Studie über „Familiäre Werte in der Türkei“ (Türkiye'de Aile Değerleri Araştırması) wurde im Jahr 2010 von dem Türkischen Familienministerium durchgeführt und die Ergebnisse unter anderem nach dem Grad des Bildungsabschlusses aufgeschlüsselt. ${ }^{13} 15,4 \%$ der Befragten mit Universitätsabschluss bejahen das Recht auf ledige Elternschaft, während lediglich $6,9 \%$ der Befragten mit Volksschulabschluss dieser Aussage zustimmen. Insgesamt 82,4 \% der Befragten stehen der ledigen Elternschaft ablehnend gegenüber. $\mathrm{Zu}$ den Ergebnissen zu dieser Aussage wird vermerkt, dass die Haltung der Gesellschaft

10 Vgl. ebd., S. 271-275.

11 Vgl. Kalsky 2003, S. 234.

12 Vgl. Internetquellen: Jelen 2011, S. 311.

13 Topçuoğlu 2010. 
$\mathrm{zu}$ diesem Thema nach wie vor traditionell ist und das Elternsein mit ehelicher Beziehung verbunden wird. ${ }^{14}$ Die Aussage, dass das Alltagsleben unter Berücksichtigung der religiösen Vorschriften erfolgen solle, wird von 70,3\% der Befragten bejaht und von 11,9 \% der Befragten verneint. Je höher der Bildungsabschluss der Befragten war, desto häufiger wurde diese Aussage verneint. ${ }^{15} \mathrm{Da}$ die Studie quantitativ erarbeitet wurde, bleibt die Frage offen, aus welchen Umständen oder Faktoren heraus sich gegensätzliche Antworten ergeben.

Einen generellen Überblick über die Religiosität der Muslime in Österreich liefert der Zwischenbericht aus dem Jahr 2013 des Instituts für Islamische Studien unter der Projektleitung von Ednan Aslan und Erol Yıldız. ${ }^{16}$ Bei der empirischen Untersuchung geht es vor allem darum, das Phänomen muslimischer Religiosität in verschiedensten Facetten abzubilden. Die Intention, möglichst differenzierte Betrachtungen zu gewinnen, lag daran, dass in Österreich eher der Eindruck bestehe, dass es sich um eine homogene Gruppe von Gläubigen handle. ${ }^{17}$

Für die qualitativen Interviews wurden Personen, die nach Österreich eingewandert sind, Angehörige der zweiten und dritten Generation sowie zum islamischen Glauben konvertierte Personen unterschiedlichster Herkunft gewählt, mit der einzigen Voraussetzung, dass sie sich selbst als Muslim/Muslimin bezeichneten. ${ }^{18}$ Interviewt wurden Personen ab 17 Jahren. Bei der Auswahl der Interviewpartner wurde darauf geachtet, die gleiche Anzahl an Personen mit niedrigem, mittlerem und höherem Bildungsniveau einzubeziehen. ${ }^{19}$

Im Rahmen der Analyse des empirischen Materials konnten fünf differenzierte Typen religiöser Alltagspraktiken ermittelt werden, nämlich: Pragmatischer Umgang mit Religion, religiöse Emanzipation, Rückzug in die Religion, säkularisierter Umgang mit Religion sowie Distanzierung und Abwendung von Religion. $^{20}$

Im Zusammenhang mit dem Typus pragmatischer Umgang mit Religion wird erwähnt, dass die Verrichtungen religiöser Tätigkeiten an den jeweiligen Kontext angepasst würden. ${ }^{21}$ Ein interessantes Ergebnis der Studie ist der Typus religiöse Emanzipation in der ein selbstbestimmter und mündiger Umgang mit der Religion beobachtet wird, der durchaus vom traditionellen Kanon „richtiger“ Religionsausübung abweicht. ${ }^{22}$

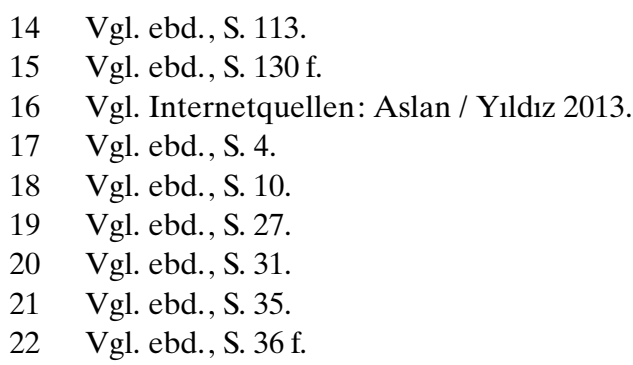




\section{Handbuchorientiertes Islamverständnis der Muslime türkischer Herkunft}

Zunächst soll ein Blick auf die Entwicklungen und Charakteristika des Islam geworfen werden, die den Rahmen für die Fokussierung des hier betrachteten Phänomens der muslimischen Religiosität im türkischen Alltag bilden. Dies ist insofern wichtig, als das Religionsverständnis der Interviewpartnerinnen einerseits vom dominierenden Islamverständnis der Muslime türkischer Herkunft abweicht, und andererseits dieser Mainstream als ursprünglicher Bezugspunkt ihres eigenen Religionsverständnisses darstellt.

Historisch betrachtet hängt die große Zahl der Muslime türkischer Herkunft, sowohl in Deutschland als auch in Österreich, mit der Arbeitsmigration aus der Türkei zusammen. ${ }^{23}$

Laut der Hochrechnungen des Zwischenberichts „Muslimische Alltagspraxis in Österreich“ sind 6,8 \% (573.876) der österreichischen Bevölkerung muslimischen Glaubens. 114.119 der Muslime in Österreich wiederum haben die türkische Staatsbürgerschaft, womit Türkischstämmige den größten Teil der muslimischen Bevölkerung Österreichs darstellen. ${ }^{24}$

Der Religionspädagoge Ednan Aslan ist der Ansicht, dass die islamischen Organisationen in Österreich vorwiegend herkunftsorientiert agieren und es neben den 60 ,ansässigen“ ATIB-Moscheen (Türkisch Islamische Union für kulturelle und soziale Zusammenarbeit in Österreich) noch 135 weitere türkische Moscheen im Land gibt. ${ }^{25}$

Ähnliche Ansichten zur Verbreitung des Islams als Resultat der Arbeitsmigration nach Europa vertritt auch der Religionspädagoge Rauf Ceylan aus Deutschland. ${ }^{26}$ Die Ergebnisse von Ceylan und Aslan deuten darauf hin, dass sich durch die Arbeitsmigration der MuslimInnen türkischer Herkunft nach Deutschland und Österreich auch ein gewisses religiöses und kulturelles Denkmuster importiert wurde, dessen Merkmale im Folgenden beleuchtet werden:

Der türkische Theologe Mustafa Öztürk und der deutsch-türkische Theologe Rauf Ceylan weisen im Islamverständnis der Muslime türkischer Herkunft besonders auf den Volksislam - und damit eine Art „Volksfrömmigkeit“ - hin. ${ }^{27}$ Öztürk teilt dabei die Volksfrömmigkeit in zwei Typen. Während es in der ersten Typologie vielmehr um Heiligenverehrung und außerislamische Inhalte geht, bezieht sich die zweite Typologie auf einen Volksislam sui generis. Dieser Typ wird von Öztürk auch als ein konservativer Islam beschrieben, in welchem außerislamische Inhalte und Erfindungen keinen Platz haben. Er fügt hinzu, dass bei

23 Vgl. Aslan 2013, S. 59.

24 Vgl. Internetquellen: Aslan / Yildiz 2013, S. 21.

25 Vgl. Aslan 2013, S. 63.

26 Vgl. Ceylan 2008, S. 48-50.

27 Vgl. ebd.; vgl. auch Internetquellen: Öztürk. 
beiden Typen grundsätzlich ein Desinteresse an theologischen Inhalten, dafür jedoch ein starkes Interesse an der Einhaltung des Islam in Bezug auf die Form, also eine blinde Nachahmung und eine starke Abhängigkeit von Ritualen, bestehe. ${ }^{28}$

Auf einen weiteren relevanten Aspekt im Religionsverständnis der Muslime türkischer Herkunft weist Ramazan Altıntaş hin. Der türkische Theologe Ramazan Altıntaş ist der Ansicht, dass das Islamverständnis vieler Menschen vor allem durch die leicht zugänglichen religiösen Handbücher geprägt ist und diese von Muslimen türkischer Herkunft ebenso als Orientierungsrahmen für die religiöse Praxis herangezogen werden. Diese Tendenz vergleicht er mit dem Konsum von Fast-Food. Nach Altıntaş verursachen die religiösen Handbücher, wie Fastfoodgerichte, langfristig gesundheitliche Schäden: viele Handbücher wurden von religiös unwissenden Menschen geschrieben, die dem religiösen Denken der Gläubigen schaden. ${ }^{29}$

Eine ähnliche Auffassung vertritt der türkische Theologe Necdet Subaşı. Seiner Ansicht nach formt sich die Religiosität der türkischen Gesellschaft durch eine Art von Handbuch-Kultur und orientiert sich vor allem an der zentralen Frage „Was ist eigentlich verboten?" 30

Auch der türkische Theologe Hayri Kırbaşoğlu weist auf den enormen Stellenwert der religiösen Handbücher im Leben der Menschen hin und beklagt, dass die Auswirkungen dieser Handbücher von den Verfassern ignoriert werden obwohl Millionen von Menschen diese Bücher als Grundwerke für ihr Glaubensverständnis und als Anleitung für ihre religiöse Praxis betrachten. Seiner Meinung nach können die Inhalte dieser Bücher bei den Menschen aufgrund der sehr strengen Vorschriften psychopathologische Wirkungen auslösen, ${ }^{31}$ da es beispielsweise um Fragen ginge, ob eine Gebetswaschung und ein rituelles Gebet ungültig werden können, wenn ein sehr kleiner Hautfleck ungewaschen bleibt. In Folge entstünde eine Art Alltagsparanoia.

Weiters konstatiert er, dass viele dieser Handbücher ein „,verengtes Religionsverständnis“ aufweisen und besonders die Inhalte über die Fünf Säulen des Islam sehr detailliert ausgeführt werden. ${ }^{32}$ Der Theologe Ilhami Güler aus der Türkei macht auf die Relevanz der religiösen Handbücher unter einem weiteren Aspekt aufmerksam. Aus einer ungewollt distanzierten Haltung zum Koran, da der Koran ja in arabischer Sprache offenbart wurde, folgert Güler, dass eine große Masse der Menschen sich den Islam eben vor allem aus den religiösen Handbüchern aneignet. ${ }^{33}$

\footnotetext{
28 Vgl. Internetquellen: Öztürk.

29 Vgl. Internetquellen: Altıntaş.

30 Vgl. Subaşı 2008, S. 132 f.

31 Vgl. Kırbaşoğlu 2002, S. 122.

32 Vgl. ebd., S. $110 \mathrm{f}$.

33 Vgl. Güler 2005, S. 30 f.
} 
Auch der türkische Kolumnist und Taha Akyol betont, dass viele Inhalte durch die Handbücher im Namen der Religion weitergegeben würden, die seiner Meinung nach auf veralteten Sprüchen und Traditionen basieren. Hier bezieht er sich beispielsweise auf das Händeschütteln zwischen Mann und Frau, das in vielen religiösen Handbüchern in religiöser Hinsicht als „verboten“ festgeschrieben wird. ${ }^{34}$

Weitere Kritikpunkte seitens der türkischstämmigen Theologen und Professoren unterschiedlicher Disziplinen ${ }^{35}$ beziehen sich auf Inhalte wie „Angst“, „Hölle“, „Bestrafung im Grab“ und ganz wesentlich auf die patriarchalische Ausformung der Inhalte über die Stellung der Frau. ${ }^{36}$

Das verbreitete Ansehen der religiösen Handbücher ist vor allem im Hinblick auf den Orientierungsrahmen für die muslimische Frau relevant. Die Frau wird in diesen Büchern grundsätzlich als mit dem Mann vor Gott gleichgestellt beschrieben, allerdings sei der Mann aufgrund seines Geschlechtes als Oberhaupt zur Versorgung seiner Familie verpflichtet und die Frau daher für die häusliche Sphäre und für die Erziehung ihrer Kinder zuständig.

Die Frau sei aufgrund ihrer psychologischen und körperlichen Eigenschaften vom Mann unterschieden, wobei von Gott nicht die Überlegenheit des Mannes bezweckt sei. ${ }^{37}$ In diesem Zusammenhang wird auch die Bildung der muslimischen Frau thematisiert, jedoch ebenfalls nur im Rahmen von Ehe und Familie, denn die Frau sei für die optimale Erziehung ihrer Kinder verantwortlich und müsse sich vor allem diesbezüglich Wissen aneignen. Die dafür nötigen Wissensbereiche seien aber auf Religion, Haushaltsführung und Kindererziehung beschränkt. $^{38}$

Der Theologe Mustafa Öztürk erwähnt einerseits die idealisierte Mutterrolle als Vorbild einer heiligen Frau und andererseits die bis heute gültige Diffamierung von Frauen als „Unruhestifterinnen“ sobald sie ihre häusliche Sphäre verlassen. ${ }^{39}$

34 Vgl. Akyol 2008 S. 256-258.

35 Die Relevanz der religiösen Handbücher zeigt sich auch darin, dass Professoren aus unterschiedlichen Instituten zum Thema Religion Bücher bzw. Artikel verfasst haben. Beispielsweise plädierte Fehmi Yavuz für die Entwicklung einer laizistischen Ethik in der Gesellschaft in der Türkei. Fehmi Yavuz (gest. 1991), Dekan am Institut für Politikwissenschaften (1958-1960) und Bildungsminister (1960) ging davon aus, dass viele Handbücher unter dem Einfluss der mystischen Bücher geschrieben wurden und diese Bücher das Religiositätsverständnis der Menschen in der Türkei fast massenhaft geformt haben. Vgl. Yavuz 1961, S. $121 \mathrm{f}$.

36 Vgl. Yavuz 1961, S. 121-124.

37 Vgl. ebd., S. 47.

38 Vgl. ebd., S. 21.

39 Vgl. Öztürk 2011, S. 111-117. 
Um die geschlechtsstereotypen Anweisungen für die muslimische Frau in den Handbüchern zu veranschaulichen, führe ich im Folgenden einige Inhalte ausgewählter Frauenhandbücher konkreter aus.

Das Frauenhandbuch „İahl Kadın İlmihali Ansiklopedisi“ (übersetzt: Kommentierte Frauenhandbuch-Enzyklopädie) erschien im Jahre 1993. Dieses Buch, das nach wie vor überall zugänglich ist, wurde vom Ehepaar Uysal geschrieben. Hier wird die Frau zwar als Geschöpf Gottes gelobt, aber in erster Linie im Zusammenhang mit ihrem Muttersein und als Frau ihres Mannes erwähnt. Mit der Aussage des Propheten Muhammad „Das Paradies liegt zu den Füßen der Mütter“40 wird in diesem Buch der ,positive“ Stellenwert der Frau hervorgehoben. ${ }^{41}$ Im Rahmen der islamischen Gesellschaft gäbe es auch Berufe, die für Frauen geeignet seien. Dafür solle die Ausbildung aber entweder innerhalb ihres eigenen Geschlechtes - also nur unter Frauen - erfolgen oder, wenn dies nicht möglich sei, müsse die Frau sich islamkonform kleiden und sich in der Bildungseinrichtung, in der ihre Ausbildung gemeinsam mit Männern stattfindet, „unauffällig"verhalten, wobei der Blickkontakt der Studentin mit dem Professor vermieden werden solle. ${ }^{42}$ Grundsätzlich wird das Arbeiten der Frau aber als Belastung wider ihre „Natur“ interpretiert ${ }^{43}$ und als etwas, von dem sie durch ihre Religion befreit sei, da die finanzielle Verantwortung für die Familie vom Ehegatten getragen werden müsse. ${ }^{44}$

Das Handbuch von Hamdi Döndüren wird hier angeführt, da es auch den Abonnenten der türkischen Zeitschrift Yeni Şafak im Jahre $2011^{45}$ geschenkt wurde und diese Zeitschrift weit verbreitet ist. Der Verfasser des Buches Hamdi Döndüren ist als Professor an einer theologischen Fakultät in der Türkei tätig. Er schreibt, dass auch die Frau als Teil der Gesellschaft fungiere und zu Bildungszwecken ihre Wohnung mit der Erlaubnis des Erziehungsberechtigten (ihres Vaters) verlassen darf. Bei verheirateten Frauen gelte die Zustimmung des Ehemannes. $^{46}$

Sein Handbuch enthält auch ein Kapitel über die Vorschriften zum gemeinsamen Leben für Männer und Frauen. Die Frau sei im Islam nicht in ihre Wohnung eingesperrt. Männer und Frauen sollen jedoch, nach Döndüren, getrennt zu unterschiedlichen Zwecken wie Unterhaltung, Bildung, Gottesdiensten etc. zusammenkommen, da auch in der Zeit des Propheten die Geschlechtertrennung beachtet wurde. Männer und Frauen bilden nach Döndüren getrennte Gruppen, und der Prophet nahm sich extra Zeit für die Frauen, da diese Frauen sonst ihre

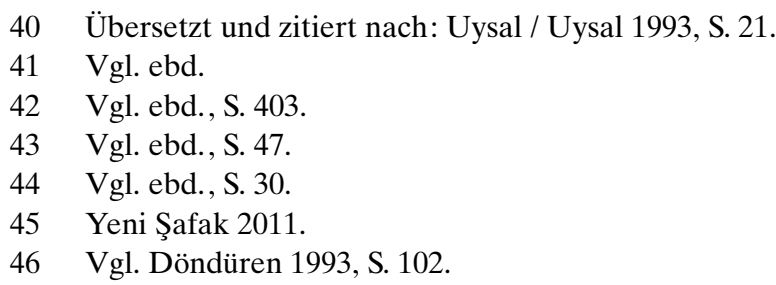


Fragen nicht an den Propheten hätten richten können, weil der Prophet stets von Männerpublikum umgeben war. ${ }^{47}$

Die Frau solle auch an ihrem Ausbildungsort, bei ihrer Arbeit und bei sonstigen diversen Tätigkeiten ihre Blicke unter Kontrolle halten, sich islamkonformunauffällig, locker bekleiden, nur der Notwendigkeit gemäß sprechen und ein Treffen mit einem Mann vermeiden. Lange Gespräche könnten ebenfalls Unruhe (fitna - Enstehung der sexuellen Anziehung) verursachen. ${ }^{48}$

Die dargestellten Inhalte scheinen trotz der hohen Auflage (41. Auflage) nach wie vor aktuell zu sein. So schreibt der Theologe Faruk Beşer, Professor aus der Türkei, in seinem aktuellsten Buch Hanımlara Özel Fetvalar (übersetzt: Spezielle Rechtsgutachten für die Frauen) bezüglich der Fragestellung, ob eine Frau bei Konferenzen vor einem männlichen Publikum präsentieren dürfe, dass ein weiblicher Auftritt vor Männern verboten sei. Er antwortet mit einer Gegenfrage, nämlich ob es wirklich notwendig sei, vor einem männlichen Publikum zu präsentieren. Er ergänzt seine Antwort aber damit, dass dies genauer diskutiert werden müsse. $^{49}$

Hinsichtlich der Frage, ob Frauen Arabisch-Unterricht bei einem männlichen Lehrer in Anspruch nehmen dürfen, kategorisiert Beşer das Lernen der arabischen Sprache als gemeinschaftliche und nicht als persönliche Pflicht und stellt fest, dass sich jedermann vorerst das Pflichtwissen aneignen müsse. Da es sich nach Beşer bei der Aneignung der arabischen Sprache nicht um Pflichtwissen handle, muss beachtet werden, ob gewisse Voraussetzungen wie z. B. die Anwesenheit einer dritten Person, vorschriftenkonforme Bekleidung und Nicht-Vorhandensein von Fitna (Entstehung der sexuellen Anziehung) in Hinblick auf die Unterrichtsstunde erfüllt sind. Die Lehr-Situation müsse nach Beşer sehr streng gehandhabt werden. Der Lehrer solle seine Schülerin/Studentin nicht anschauen, ein Vorhang sei die bessere Option. Trotz seines Bezuges zur hanafitischen Rechtsschule, dass der Mann von einer Frau Gesicht und Hände sehen dürfe, argumentiert Beşer, dass langer Blickkontakt Fitna verursachen könne und daher der Unterricht am besten ganz vermieden werden sollte. ${ }^{50}$

Viele Leserinnen und Leser akzeptieren die in den Handbüchern ausgeführten Verpflichtungen und Verbote, da die meisten Verfasser eine theologische Ausbildung vorweisen und sich in ihren Argumentationen auf die Primärquellen (Koran und Sunna) des Islam beziehen.

Beispielsweise wird die Aussage des Propheten über das Verbot des zweiten Blickkontakts als eine Beweisquelle für eine empfohlene Geschlechtertrennung auch in Bildungseinrichtungen herangezogen. Handbuchverfasser Hasan Calış-

47 Vgl. ebd., S. $114 \mathrm{f}$.

48 Vgl. ebd., S. 119-121.

49 Vgl. Beşer 2013, S. 135 f.

50 Vgl. ebd., S. $109 \mathrm{f}$. 
kan bezieht sich in diesem Zusammenhang auf die folgende Überlieferung des Propheten Muhammad:

Jārir said: I asked the Apostle of Allah (may peace and upon him) about an accidental glance (on a woman). He said: Turn your eyes away. Buraidah reported the Apostle of Allah (may peace be upon him) as saying to 'Alī: Donot give a second look, 'Alī, (because) while you are not to blame for the first, you have no right to the second. ${ }^{51}$

Auch das Handbuch Evlilik ve Aile Hayatı (übersetzt: Ehe- und Familienleben) von Hasan Calışkan wurde von der türkischen Zeitschrift Zaman den Abonnenten im Jahre 2004 als Geschenk übermittelt. Dieses Detail zeigt wiederum die Relevanz der Verbreitung der Inhalte über die Türkei hinaus, leben doch viele der Abonnenten der Zeitschrift mittlerweile in der EU. ${ }^{52}$

Das durch religiöse Handbücher oder Volksfrömmigkeit geformte Islamverständnis scheint im Leben der interviewten Frauen interessanterweise keinen Platz zu haben. Im Zuge der Analyse der Interviewgespräche konnte allerdings festgestellt werden, dass die jungen Frauen von einem konservativen oder eben einem handbuchorientierten Islamverständnis ausgegangen waren, diese Art der Auslegung jedoch im Laufe ihrer Studienzeit nach einer kritischen Auseinandersetzung mit den erlernten Inhalten modifiziert haben. Die Adaption religiöser Werte und kultureller Haltungen bildet in gleicher Weise wie der Studienabschluss oder die Berufstätigkeit einen bedeutsamen Faktor in Bezug auf die gesellschaftliche Positionierung und die Emanzipationsbestrebungen der Interviewpartnerinnen.

\section{Ausgewählte Ergebnisse}

Das Forschungsinteresse zu gebildeten Frauen türkischer Herkunft formte sich zunächst aufgrund der stereotypisierten Handhabung der muslimischen Frau im öffentlichen Diskurs, basierend auf dem Umstand, dass sich tatsächlich immer noch viele muslimische Frauen innerhalb ihrer traditionellen kulturellen Werte bewegen. Um dies zu konkretisieren: Laut Angaben des Österreichischen Integrationsfonds aus dem Jahr 2010 war die Zunahme der muslimischen Bevölkerung zwischen den Jahren 2001 und 2009 in wesentlich stärkerem Ausmaß auf eine höhere Geburtenrate zurückzuführen als auf Zuwanderung. ${ }^{53}$ Ein davon abweichendes Frauenbild ist allerdings bei jungen Akademikerinnen zu beobachten, die über einen Universitätsabschluss verfügen und auf ihre persönliche und berufliche Qualifizierung Wert legen. Durch meine besondere Rolle, sowohl als

\footnotetext{
51 Abū Dāwūd, Sunan: Kitāb al Nikāḥ, Chapter 710/2143 f.

52 Vgl. Calışkan 2004.

53 Vgl. Internetquellen: Marik-Lebeck 2010, S. 5-9.
} 
Forscherin als auch als Mitglied der Community, bot sich mir ein Feldzugang, der Personen nicht-türkischer Herkunft möglicherweise nicht in dieser Weise offen steht.

Die geführten Interviews zielten darauf ab, Erkenntnisse über die Bedeutung der universitären Bildung für das Leben und Denken der Frauen im Alter von 25 bis $35 \mathrm{zu}$ gewinnen.

Die Hauptfrage bezog sich dabei auf die Rolle der Religion im Leben der Interviewpartnerinnen bzw. auf die mögliche Transformation von Religiosität durch höhere Bildung.

Die offenen Leitfadenfragen in den Interviewgesprächen deckten einen vielfältigen Themenradius ab: von der Bildungsgeschichte der Frauen, ihrem biografischen Hintergrund und ihrem persönliches Frauenbild über die Vereinbarkeit von Beruf und Mütterlichkeit bis hin zu ihrer persönlichen Positionierung zur Stellung der Frau im Islam. Des Weiteren wurden allgemein das persönliche Outfit und die generelle Bedeutung von Religion, unabhängig von einer regelmäßigen Praxis, in den Interviewgesprächen thematisiert. ${ }^{54}$

Die Auswertung der Interviews erfolgte unter Anwendung der Systemanalyse von Ulrike Froschauer und Manfred Lueger. ${ }^{55}$ Die Methode zeichnet vor allem aus, dass die Auslegung der Textausschnitte unter Einbeziehung mehrerer Interpretationsgruppen erfolgt, um eine ausführliche, differenzierte und objektive Analyse der ausgewählten Textausschnitte zu gewährleisten. Alle TeilnehmerInnen, die bei der Analyse der gewählten Textausschnitte mitwirken (Interpretationsgruppen), müssen mit der Methode der Systemanalyse, die bei der Auswertung insbesondere gewissen Voreingenommenheiten seitens der Interviewenden entgegenwirken soll, vertraut gemacht werden. Für eine präzise Analyse wird der gleiche Interviewausschnitt von mehreren Interpretationsgruppen analysiert.

Die Besonderheit bei der Systemanalyse ist, dass das Analysieren von größeren Textmengen möglich wird, indem man sich in diesem Vorgang an der ErschlieBung ,prozessdynamischer Aspekte komplexer und intern hochqualifizierter sozialer Felder ${ }^{156}$ orientiert. Somit ermöglicht die Systemanalyse die Auslegung einzelner größerer oder vollständiger Textausschnitte, wobei der Analysefokus sich nicht auf manifeste, offenkundige Stellen reduziert, sondern durch die extensive Auslegung verschiedene Strukturierungsphänomene jenseits des unmittelbaren Kontextes berücksichtigt werden können. ${ }^{57}$

Die Auswertung der Interviews gestaltet sich durch den Zirkel einer schrittweisen Interpretation der ausgewählten Textausschnitte und der darauf erfolgenden permanenten Revidierung der Annahmen. Dabei müssen die Textaus-

54 Vgl. Dursun 2015, S. 102-107.

55 Vgl. Froschauer / Lueger 2003.

56 Ebd., S. 146.

57 Vgl. ebd., S. $95 \mathrm{f}$. 
schnitte so gewählt werden, dass maximale strukturelle Variation gewährleistet ist, weshalb auch jene Stellen berücksichtigt werden, die den bis dahin gefassten Annahmen am deutlichsten widersprechen. Eine weitere Besonderheit dieser Methode ist, dass bei der Auswahl der Interviewpartnerinnen Kriterien der Unterschiedlichkeit, der Ähnlichkeit sowie der Grenzen des sozialen Systems berücksichtigt werden müssen und solange nach weiteren Interviewpartnerinnen gesucht wird - wobei parallel dazu das Analyseverfahren fortgesetzt werden muss - bis die theoretische Sättigung erreicht ist.

Nachdem die ersten Thesen (Forschungsergebnisse) durch die von den Interpretationsgruppen durchgeführten Interpretationen eine gewisse Stabilität erlangt haben, dürfen diese als vorläufig abgeschlossen betrachtet werden (theoretische Sättigung). ${ }^{58}$

Die Analyse der Interviewausschnitte mittels mehrerer Interpretationsgruppen erfolgt durch eine strukturierte Vorgehensweise. Zunächst wird der Textausschnitt inhaltlich zusammengefasst. ${ }^{59}$ In der ersten Etappe der Analyse ist vorgesehen, dass der Textausschnitt hinsichtlich seines Äußerungskontextes ausgewertet wird. Der Äußerungskontext, der sich inhaltlich in „Textrahmen“ und „Lebenswelt“" unterteilt, hat die Aufgabe, sich in die Rolle der Textproduzenten hineinzuversetzen. Der Textrahmen bezweckt die Intention des Textproduzenten gegenüber dem/der Interviewenden zu deuten. ${ }^{60}$ Unter der Kategorie Lebenswelt gilt es den die Interviewpartnerin prägenden kulturellen, sozialen und religiösen Kontext in den Blick zu bekommen, um zu analysieren. ${ }^{61}$

In der zweiten Etappe der Analyse befassen sich die Interpretationsgruppen mit dem Wirkungskontext aufgrund des ermittelten Äußerungskontextes. Der hypothetische Wirkungskontext 1 beschäftigt sich mit der Frage, welche Folgen sich aufgrund des ermittelten Äußerungskontextes für die unmittelbaren Handlungsstrukturierungen der befragten Person ergeben könnten. Der hypothetische Wirkungskontext 2 berücksichtigt die Gesamtdynamik des sozialen Systems mit seiner Auswirkung auf das Zusammenspiel mit anderen Subsystemen oder AkteurInnen. Ebenfalls untersucht man im Text, ob Prozesse der Abgrenzung, Stabilisierung oder Verbindung zu beobachten sind. ${ }^{62}$

Um Nachfragen oder Unterbrechungen im Analyseverfahren durch eigene Notizen zu vermeiden, ist es empfehlenswert auch die Interpretationsgespräche auf Tonband aufzunehmen und gleichzeitig auch die Kommentare während der Sitzungen stichwortartig und nach tabellarischer Gliederung zu notieren. Ich übernahm während den Interpretationssitzungen lediglich die Aufgabe für die Moderation und die Führung der stichwortartigen Aufzeichnungen. Für das ge-

\begin{tabular}{ll}
\hline 58 & Vgl. ebd. \\
59 & Vgl. ebd., S. 155. \\
60 & Vgl. ebd. \\
61 & Vgl. ebd., S. 151. \\
62 & Vgl. ebd., S. $151 \mathrm{f}$.
\end{tabular}


samte Analyseverfahren wurden Personen aus unterschiedlichen Erfahrungsfeldern involviert. Besonders bei frauenspezifischen Textausschnitten und bei Textausschnitten über die Religion wurde darauf Wert gelegt, sowohl männliche als auch weibliche Interpretationssitzungen zustande zu bringen. Auch Personen unterschiedlicher Religionszugehörigkeiten und religionskritische Personen wurden in die Interpretationsgruppen einbezogen.

Im Folgenden werden anhand der Erzählungen der interviewten Frauen implizite und explizite Modifikationshinweise angeführt, die einen wesentlichen Bezug zum oben genannten, handbuchorientierten Islamverständnis zeigen. Diese Modifikationshinweise können auch als eine feministische bzw. gendergerechte Lesart des Islam betrachtet werden.

In den Interviews wurde nicht explizit nach Änderungen hinsichtlich der religiösen Einstellungen gefragt. Dennoch kamen fast alle Interviewteilnehmerinnen von sich aus darauf zu sprechen. ${ }^{63}$

\subsection{Islamischer Kleidungsstil - Sinnzuschreibung und Einordnung}

Der erste Modifikationshinweis, der hier nun ausgeführt wird, bezieht sich auf die Bedeutung des islamischen Kleidungsstils und kommt von Ip $3^{64}$, Wirtschaftsinformatikerin mit Masterabschluss. Sie ist 30 Jahre alt, verheiratet, karenziert. Ip3 wurde in Wien geboren. Ihre Eltern kamen als Gastarbeiter nach Österreich, um eine gewisse Zeit in Österreich zu arbeiten und danach wieder in die Türkei zurückzukehren. Sie wuchs bei ihren Großeltern auf und kam erst nach ihrem Hauptschulabschluss nach Österreich, da der Rückkehrwunsch der Eltern sich auflöste.

Bezogen auf die Frage, auf welche Punkte Interviewpartnerin 3 (Ip3) bei der Wahl ihres Outfits achtet, erzählte sie, dass sie in ihrer Studienzeit die Einstellung hatte, sich streng islamisch kleiden zu müssen. (Dieses Outfit charakterisiert sich grundsätzlich durch ein Kopftuch, mit dem alle Haare bedeckt werden, und körperunbetonte Gewandung.) Bis zu ihrem Studienabschluss achtete sie auf ihr islamisches Outfit, das während ihrer Studienphase einen zentralen Aspekt ihres

63 Die Interviews wurden zwischen Mai und Oktober 2014 durchgeführt. Jedes einzelne Interview dauerte zwischen 45 Minuten und zwei Stunden. Jedes Interview wurde vollständig transkribiert und anonymisiert. Aus Datenschutzgründen wurde die vollständige Transkription nicht der Dissertationsarbeit zur freien Handhabe zur Verfügung gestellt. Aus diesem Grund behält sich die Interviewerin das Recht vor, nur die anonymisierten Transkripte aus ihrer Dissertationsarbeit als Quelle anzugeben.

64 Statt für die Interviewpartnerinnen einen Namen zu erfinden, wurden alle Interviewgespräche anonymisiert und jeweils chronologische Nummern vergeben. Das bedeutet also, dass jene Interviewpartnerin die Benennung „Ip1“ bekam, mit der ich das erste Interviewgespräch durchführte. 
Lebens bildete. In Hinblick auf ihr Erscheinen zum Interview ohne Kopftuch, erzählte sie von der Veränderung ihrer Einstellung zu Bekleidung generell im Kontext ihrer Berufstätigkeit in einer männerdominierten Branche.

Die gelernte Auffassung zur islamischen Kleidung, interpretiert sie dahingehend, dass dies die Frauen möglicherweise vor Blicken der Männer schützen solle.

Allerdings wird ihr während ihrer Berufstätigkeit bewusst, dass die Männer sie gar nicht anschauen und an ihrem Arbeitsplatz effektives Arbeiten im Vordergrund steht. Die individuelle Auslegung der Ip3 zeigt, dass sie bezüglich „,der Nichteinhaltung des islamischen Outfits" kein schlechtes Gewissen hat, da die Männer sie sowieso nicht anschauen und sie dadurch den Sinn der Tradition auch ohne ein Kopftuch als erfüllt betrachtet. Um in ihrer Arbeit effektiv Leistungen zu erzielen, bevorzugt sie einen Kleidungsstil, der sie dabei nicht stört.

\section{S: Welche Punkte sind Dir bei deinem Outfit wichtig?}

Ip3: Also es ist so bei mir eine Wende gewesen... früher wie ich studiert habe, habe ich mir gedacht, dass ich mich religiös gesehen sehr streng anziehen muss... und ich habe das Gefühl gehabt, dass es ist das Wichtigste in meinem Leben ist... nur dieser Gedanke hat sich zum Beispiel seit dem ich arbeite aufgelöst... ich denke, man respektiert mich wirklich mit meiner Intelligenz und nicht wegen meinem Gewand, das ich anziehe .. und ich finde in Österreich ... steht Outfit wirklich nicht an erster Stelle [...]

S: Wie kam es zu dieser Wende [..]?

Ip3: [...] in dem Arbeiten [...] ich bin in einer Branche, wo ich mit mehreren Männern zu tun habe ... und ich habe das Gefühl gehabt, sie schauen nicht einmal (lacht), was man an hat...es ist wirklich egal in der Firma [...] also wichtig war für mich, dass ich wirklich fünf Mal am Tag bete, dass ich faste [...]

Ip3: Wenn ich mich schon in der Firma im Unternehmen bewiesen habe... glaube ich..ja, ich will jetzt nicht übertreiben (lacht) ich kann natürlich nicht als Hippe auftreten, aber es ist nicht wichtig was ich anhabe.. außer der erste Eindruck macht etwas aus... das ist auf jeden Fall zum Beispiel bei der Bewerbung wichtig... aber sonst ${ }^{65}$ : bin ich lockerer, ich trage auch Jeans und T-Shirts und es juckt nicht so (lacht)... ${ }^{66}$

\subsection{Schönheitsideale}

Ein weiterer Modifikationshinweis hinsichtlich religiöser und kultureller Praxis bezieht sich auf den Umgang mit religiösen Vorschriften. Ip4 kam in Wien auf die Welt. Ihre Eltern heirateten in der Türkei als sie 17 Jahre alt waren und kamen nach der Eheschließung nach Wien. Zwischen ihrem ersten und vierten Lebensjahr blieb Ip4 bei ihrer Großmutter in einer kleinen Ortschaft in Mittelanatolien, weil ihre Eltern in Wien arbeiteten und Rückkehrwünsche in die tür-

\footnotetext{
65 „hm:=lang ausgesprochen“.

66 Dursun 2015, S. 196 f.
} 
kische Heimat hegten. Ip4 wuchs ab ihrem vierten Lebensjahr in Wien auf und absolvierte in Wien das Jus-Studium. Sie ist ledig und 29 Jahre alt.

Im Zuge der sehr ausführlichen Erzählungen der Ip4 über die religiöse Erziehung kam auch ihre jetzige religiöse Einstellung im Vergleich zum früheren religiösen Erleben zur Sprache.

Sie lernte den Islam wie ein kompaktes Paket kennen, das sie als Ganzes zur Kenntnis nehmen und in ihrem Leben umsetzen sollte. Ip4 dachte früher, dass sie nicht fünf Mal am Tag beten könne, wenn sie grundsätzlich keine Kopftuchträgerin sei. Mittlerweile ist sie der Ansicht, dass das Gebet zu den religiösen Vorschriften gehört, die jeder Muslim zu befolgen hat, unabhängig vom Aussehen der Person. Beten und danach ohne Kopftuch spazieren gehen oder schwimmen schließt sich für sie nun nicht mehr aus. Ihre religiösen Einstellungen haben sich geändert, was ihr zufolge jedoch nicht an ihrem Studium, sondern generell an der Änderung ihrer Denkweise liegt. Ihre Haltung zu angeblichen Verboten und religiösen Argumentationen generiert sie nun durch eigenständige Reflexion und Recherche; bei Unklarheiten (z.B. bei Fragen zu Gelnägel, Tattoos, Zahnschmuck und Prophetengeschichten) wendet sie sich mitunter auch an damit vertrautere Personen.

Wie schon im gängigen Islamverständnis der Muslime türkischer Herkunft ausgeführt wurde, erkennt man auch in den Erzählungen der Ip4, dass sich ihr früheres Islamverständnis eher an der Frage „Was ist eigentlich verboten?“67 orientiert hat. Selbstständig entwickelt sie jedoch eine kritische Betrachtungsweise und begibt sich auf die Suche nach neuen theologischen Positionen. Sie erzählte beispielsweise sehr ausführlich von einer Phase in ihrer Jugend, die mit der Einhaltung strenger Regeln einherging, etwa, dass Musikhören verboten sei. Sie wurde mit der Idee erzogen, dass bei Nichteinhaltung des Verbots brutale Bestrafung im Grab drohe. Sie betonte, dass sie diese Regel zu anfangs beachtete. Die Nichtbefolgung des gelernten Gebots verursachte bei Ip4 nach einiger Zeit jedoch ein immenses Angstgefühl und psychische Probleme, die sie aber durch Vertrauenspersonen in ihrem Umfeld bewältigen konnte. Aufgrund dieser negativen Erfahrungen, fing sie an, die religiösen Gebote zu filtern und reflektiert bzw. individuell zu interpretieren.

Auch aus den Erzählungen der Ip4 kann herausgelesen werden, dass Ip4 zunächst ein durch Handbücher geformtes Islamverständnis kennengelernt hat. Der Hinweis von Kırbaşoğlu, dass die Inhalte religiöser Handbücher bei Menschen aufgrund der sehr strengen Vorschriften psychopathologische Wirkungen auslösen können, ${ }^{6}$ bewahrheitet sich in den Äußerungen von Ip4 bezüglich der Infragestellung der Gebetswaschung mit Tatoos oder Gelnägeln: Diese löste bei ihr enorme Angst und Schuldgefühle aus. ${ }^{69}$ Der durch die eigenständige Reflexion

67 Vgl. Subaşı 2008, S. $132 \mathrm{f}$.

68 Vgl. Kırbaşoğlu 2002, S. 122.

69 Vgl. ebd., S. 234. 
erfolgende Wandel wird dann aber auch an den religiösen Einstellungen der Ip4 erkennbar. Statt an den beschriebenen inhaltlichen Kriterien für die Gültigkeit einer Regel festzuhalten, versucht sie nun ihre Schönheitsideale mit ihren religiösen Einstellungen zu vereinbaren, indem sie weder auf das Gebet noch auf ihre Schönheitsideale völlig verzichtet. Stattdessen sucht sie nach Auswegen, um ihre Schönheitsideale parallel zu ihren religiösen Praktiken zu integrieren:

Ip4: [...] ich dachte, dass es nicht geht, fünf Mal am Tag zu beten, wenn man grundsätzlich kein Kopftuch trägt.. ich dachte, dass das eine dem anderen widersprechen würden.. jetzt denke ich mir aber... das rituelle Gebet ist eine Vorschrift, die jeder Muslim erfüllen muss egal wie er aussieht... ja und jetzt denke ich.. und ich kann beten und danach schwimmen gehen ja... weil das meiner Meinung nach sehr getrennt ist.. weil Anziehen ist eine Sache und das Beten ist eine andere Sache ist.. und wenn ich bete, dann habe ich diese Vorschrift erfüllt ... [...] früher habe ich gedacht, dass das nicht gehen würde.. ich dachte, dass man generell ein Kopftuch tragen muss und beten kann ... und jetzt weiss ich.. ich muss nicht... ich kann beten und ohne Kopftuch nachher spazieren gehen..

S:Du hast diesbezüglich Deine Recherchen erwähnt...

Ip4:Ja.. Wenn ich höre zum Beispiel.. wenn mir jemand sagt, dass irgendetwas ist aus jenem Grund verboten ist. .

$\mathrm{S}: \mathrm{ja}$

Ip4: Und wenn mir das irgendwie sehr suizid (Anmerkung: Sie meinte wahrscheinlich „suspekt") . vorkommt.. und .. dann schaue ich noch einmal nach... oder ich frage zu anderen Personen, wo ich weiß, dass sie sich besser auskennen... zum Beispiel..keine Ahnung.. die Gelnägel zum Beispiel

S:Wie...?

Ip4: Ja die Gelnägel

S: Aha?

Ip4: Ich hörte zum Beispiel, dass Gebetswaschung mit Tatoo überhaupt nicht möglich ist ... Ich habe Zahnstein... oder wenn ich höre, dass zum Beispiel ... wenn ich über den Propheten etwas höre, dass sie früher dies oder jenes gemacht haben ... und wenn ich es nicht weiss... dann schau ich selber nach ${ }^{70}$

\subsection{Eine katholische Kirche als Gebetsraum}

Ein weiterer individueller Modifikationshinweis religiöser und kultureller Praktiken bezieht sich auf den Raum. Ip5 ist in der Türkei aufgewachsen und kam erst zum Studieren nach Österreich, da ihr Vater als Gastarbeiter hier lebte. Ip5, ihre Geschwister und ihre Mutter kamen erst während der Studienphase von Ip5 nach Österreich. Nach dem Abschluss des Gymnasiums setzte Ip5 ihre Bildungslaufbahn in Österreich fort und beendete ihr Doktoratsstudium in Pädagogik. Bevor Ip5 und ihre Familienangehörigen nach Österreich kamen, befand sich die Familie

70 Dursun 2015, S. 199 f. 
von Ip5 in einem sunnitischen Umfeld. Sie erzählt, dass ihre Eltern zwar Aleviten sind, jedoch aufgrund des sunnitischen Umfeldes sunnitische Traditionen pflegen. Als Beispiel bezog sie sich darauf, dass sie und ihre Familienangehörigen wie sunnitische Muslime im Monat Ramadan fasten, aber den Fastenmonat der alevitischen Muslime (im Monat Muharram) nicht einhalten. Im weiteren Verlauf erzählt sie, dass sie sich weder zur Gänze als Alevitin noch als sunnitische Muslimin fühlt. Sie meint, dass sie sich ohne einen konfessionellen Bezug generell als Muslimin fühlt. Auf Fragen über ihre religiöse Praxis, die Moschee und über die „Cem Evi“ (Gebetshaus für Aleviten) antwortete sie, dass für sie religiöse Symbole unwichtig seien und dass sie sogar in einer katholischen Kirche betete, weil Gott überall sei. $^{71}$

Für Ip5 ist es selbstverständlich, in einer Kirche zu beten. Sofern Ip5 in einem westlich-christlich geprägten Land wohnt, in dem Kirchen viel einfacher im Vorbeigehen zu besuchen sind, weshalb sie eben auch für die Ausübung der eigenen religiösen Praxis in Frage kommen. In der Türkei, in einem islamisch geprägten Land mit starker Präsenz von islamischen Symbolen, wäre ihr eine solche räumliche Integration wohl nicht in den Sinn gekommen. Das Leben in einem christlich geprägten Kontext zeigt unter diesem Aspekt sehr wohl den Wandel in den religiösen Praktiken der muslimischen Religionsangehörigen.

Des Weiteren ist es für sie selbstverständlich, dass sie auf Türkisch betet. Grundsätzlich ist es sowohl im sunnitisch als auch im schiitisch geprägten Islam üblich, dass die rituellen Gebete auf Arabisch rezitiert werden, wobei Bittgebete auch in der Muttersprache gesprochen werden können. Überall auf der ganzen Welt lernen Menschen, egal welcher Muttersprache, für die Durchführung des rituellen Gebets bestimmte Suren aus dem Koran und Bittgebete des Propheten auf Arabisch auswendig. Daher verstärkt sich die These, dass Ip5 ihre traditionelle/ursprüngliche Auslegung vom kulturellen Umfeld rezipiert hat und ihre religiöse Praxis - hinsichtlich des Betretens einer katholischen Kirche und des Vollzugs ihrer Gebete in ihrer Muttersprache - eigenständig modifiziert haben könnte. Bei der Beschreibung eines bestimmten Imam erwähnt sie dessen theologisches Studium und seinen marrokanischen Migrationshintergrund. ${ }^{72}$ Diese beiden Bemerkungen könnten sowohl auf die Wertschätzung religiös-theologischer Bildung als auch auf einen innermuslimischen Wissensaustausch in der deutschen Sprache im europäischen Kontext - anstatt eines herkunftorientierten Bezuges -, hinweisen.

Vergleichen wir Ip5 s Erzählung mit den Studienergebnissen von Karakaşoglu zu den sechs Typen religiöser Orientierung (Atheistinnen, Spiritualistinnen, sunnitische Laizistinnen, pragmatische Ritualistinnen und idealistische Ritualistinnen $)^{73}$ zeigt sich hier etwas Neues, über diese Typisierung Hinausweisendes,

71 Vgl. Dursun 2015, S. 200-203.

72 Vgl. Dursun 2015, S. $201 \mathrm{f}$.

73 Vgl. Karakaşoğlu 2003. 
nämlich eine Art von Hybridität, in der sich einerseits der spirituelle Aspekt der eigenen Religion und andererseits ein Bezug zum symbolgeprägten Aufnahmeland (katholische Kirchen) vermischen.

Ip5: Als ich im Bundesland XX war, habe ich in XZ als Lehrerin gearbeitet.. es gab in diesem Institut viele Kinder mit Migrationshintergrund.. dort habe ich unterrichtet und [...] .. und da hattten wir auch eine kleine Moschee in XZ.. Dort las ich sehr oft den Koran mit einem Imam, der aus Marokko kam.. aber er studierte auch etwas in dieser Richtung ..Ja, er war ein sehr guter Imam .. und sehr offen für alle Religionen.. [...].. manchmal habe ich sogar in der Kirche gebetet. . [...].für mich ist,... wie ich gesagt habe. ..Symbole sind für mich unwichtig.. für mich ist Gott überall wo Ihn man spürt.. spüren kann.. Gott ist überall auf der Straße auch.. [...] deswegen wenn ich bisschen alleine bleiben will.. dann..[..] zum Beispiel während ich zu arbeiten gehe oder zu Bibliothek gehe, da kenn ich eine kleine Kirche gehe ich rein .. bete ich türkisch. . und Gott ist nur der Eine und überall..[... $]^{74}$

\subsection{Verschiebung persönlicher Grenzen - Modifizierung der Distanz zum männlichen Geschlecht}

Der letzte Modifikationshinweis in diesem Artikel bezieht sich auf die Verschiebung persönlicher Grenzen. Interviewpartnerin 2 (Ip2) ist 34 Jahre alt. Sie wurde in der Türkei geboren, kam aufgrund des Kopftuchverbots zum Studieren nach Wien, lebt weiterhin in Wien und ist Elektrotechnikerin. Auch sie ist verheiratet, hat ein Kind und befindet sich derzeit in Karenz. Sie eignete sich ihr religiöses Wissen in einem theologischen Mädchengymnasium an und betonte im Interviewgespräch, dass die Religiosität ihrer Eltern sie nie begeistert hat. Das religiöse Wissen aus der Imamhatip Schule (theologisches Mädchengymnasium) formte ihre religiöse Praxis bis sie nach Österreich kam.

Sowohl bereits während als auch nach dem Studium kam es bei Ip2 zur Modifizierung ihrer religiösen Praxis. Als Grund dafür nannte sie die westliche Prägung Österreichs im Gegensatz zur Türkei. Die im Westen notwendige persönliche Klärung der eigenen Grenzen führten bei Ip2 zu einer Grenzverschiebung und zur Lockerung ihrer früheren Strenge. Früher hat sie, soweit es geht, die Nähe zu Männern vermieden. Im Studium aber war sie fast nur mit männlichen Studenten zusammen. In Österreich fiel es ihr aufgrund der unterschiedlichen Kultur und des überwiegenden Männeranteils in ihrem Studium schwer über alles zu reden, weil sie die Grenzen der gegenüberstehenden Personen nicht einzuschätzen vermochte. Die darauf folgende Reflexionsphase hat sie dazu gebracht, ihre persönlichen Einstellungen zu ändern. Sie hat begonnen, mit ihren Studienkollegen über vieles zu reden, worüber sie in der Türkei bestimmt geschwiegen hätte. Früher vermied sie sogar das Händeschütteln mit Männern. In der Türkei

Dursun 2015, S. 202. 
passt sie zwar nach wie vor auf, mit wem sie worüber spricht, die Hände schüttelt sie mittlerweile aber überall. ${ }^{75}$

Aus den Schilderungen der Ip2 lässt sich vermuten, dass sich die erlebte Distanz zum männlichen Geschlecht aufgrund der geschlechtshomogenen Schulbildung in ihrem theologischen Gymnasium und der dort erfolgten religiösen Bildung ergeben hat. Ihr Bezug auf das Händeschütteln, ist ein Hinweis dafür, dass ihre Einstellung, die sie gelernt und in ihrem Leben bis in ihre Studienzeit umgesetzt hat, handbuchorientiert geprägt war. Denn auf das Händeschütteln wird in den religiösen Handbüchern - wie der türkischen Kolumnist und Journalist Taha Akyol ausführt - sehr detailliert eingegangen. Taha Akyol betont, dass viele Inhalte durch die religiösen Handbücher im Namen der Religion weitergegeben werden, ohne den Wandel kultureller und religiöser Praktiken zu berücksichtigen. $^{76}$

Durch die Wahrnehmung der unterschiedlichen Kontexte findet es Ip2 daher nunmehr in Ordnung, die persönlichen Haltungen zur Außenwelt zu ändern, um zu erreichen, dass man in dem neuen Umfeld akzeptiert und aufgenommen wird. Ein beharrendes Festhalten an den gelernten und ursprünglich islamisch gedeuteten Praktiken, wie der Einhaltung des Verbots zum Händeschütteln zwischen Mann und Frau, und die beachtete Distanz zum anderen Geschlecht verschwindet in der religiösen Haltung der Ip2. Stattdessen modifiziert Ip2 ihre religiöse und kulturelle Praxis, um sich aufgrund ihrer religiösen Einstellungen in ihrem neuen Kontext nicht zurückziehen zu müssen, was ein wesentliches Hindernis auf dem Weg zu ihren Emanzipationsidealen bedeuten würde. Insofern ergibt sich kein Bruch zur religiösen Praxis, sondern eine Berücksichtigung unterschiedlicher kontextueller Faktoren, die der Ip2 das Gefühl vermittelt, dass sie sich weiterhin innerhalb der ihr wichtigen religiösen Regeln bewegt.

Natürlich.. du lebst ja in einem Land.. wo es eigentlich.. ich sage nicht,.. dass es christlich geprägt ist. weil ich glaube nicht.. dass, alles was in der Gesellschaft ist.. von Religion geprägt ist. . besonders in Europa ist es eben eine westliche Kultur.. [...] aber wenn man in der Türkei dich sieht, dann weiß man wie eine Person ist. . [....] .. hier muss man sich zuerst Mal vorstellen. . bekannt machen, klar machen, wo deine Grenzen sind [...] stell dir vor.. ein Mädchenschule hab ich besucht.. was ich mache ist ein Studium wo nur drei Mädchen sitzen.. [..] es war ein bisschen schwierig.. über alles zu reden was ich denke.. ich wusste nicht wie sie reagieren würden.. [...] es wäre viel einfacher gewesen wenn sie so aus einer anderen Kultur aber Frau gewesen wären.. das war für mich eine andere Kultur und noch dazu Männer.. wo ich gedacht habe.. hey was kann ich machen?... was werde ich sagen?. . [...] Und diese Überlegungsphase hat mich dazu gebracht, dass ich meine Einstellung mal geändert habe.. langsam hab ich angefangen über vieles zu reden.. wo ich in der

75 Vgl. Dursun 2015, S. 186 f.

76 Vgl. Akyol 2008, S. 256-258. 
Türkei wahrscheinlich niemals gesprochen hätte (l) diese Offenheit.. [...] außerhalb der Familie hab ich zum Beispiel .. und zum Beispiel keine Hände geschüttelt. ${ }^{77}$

\section{Schlussbemerkung: Die Entwicklung reflexiver Religiosität}

Die exemplarische Auswahl der Textausschnitte zu den Themenblöcken „Islamisches Outfit/Sinn“, „Schönheitsideale“, „Kirche als Gebetsraum“, „Verschiebung persönlicher Grenzen“ - zeigt die ambivalenten Bemühungen der Interviewpartnerinnen, beide Welten (sowohl die religiöse als auch die moderne Lebensführung) auf eine gesunde und innovative Art zusammenzuführen, ohne einen Bruch mit der religiösen Tradition zu erzeugen. Noch genauer lassen sich die Ausformungen der religiösen Identitäten der Interviewpartnerinnen mit dem Ansatz der reflexiven Religiosität beschreiben. Der Religionssoziologe Gerardo Marti bezieht sich in seinem Artikel „Religious Reflexivity: The Effect of Continual Novelty and Diversity on Individual Religiosity “78 auf die Ansätze von Ulrich Beck, Peter Berger und Margaret Archer und eben deren Begrifflichkeit der „Reflexiven Religiosität":

By resourcing Archer, Beck, and Berger, I summarize a core dynamic of modern experience through the concept of „religious reflexivity.“ Reflexivity is a ubiquitous human dynamic, yet the capacity for distinctively religious reflexivity is strained to the degree that the logics and routines of religious life fail to fit the logics and routines that dominate civic, market, and state-mediated contemporary life. Here, I stress that religious reflexivity points to a deliberative and problem solving dynamic that is a distinctive and unavoidable element of contemporary religious selves. In times of greater social change, the imperative for reflexivity is made incumbent for all; moreover, the greater the degree of social change, the greater the invocation of religious reflexivity. This is not to say that religion is "rational" or that it involves a narrowly defined sense of personal „belief". Instead, the notion of religious reflexivity accentuates how individuals exercise their agency in living out their religious imperatives in the modern world. ${ }^{79}$

Basierend auf diesem Ansatz ist auch Marti der Ansicht, dass die sich permanent ändernden sozialen Strukturen einen wesentlichen Einfluss auf die individuelle Religiosität mit sich bringen. Er ist davon überzeugt, dass Religion einen Teilbereich der westlichen Modernität darstelle, was sich in Faktoren wie Globalisierung, Urbanisierung, Bildung und in den sozialen Netzwerken und Kommunikationstechnologien zeige. ${ }^{80}$

\begin{tabular}{ll}
\hline 77 & Dursun 2015, S. 188. \\
78 & Marti 2015. \\
79 & Ebd., S. 3. \\
80 & Vgl. ebd., S. 2.
\end{tabular}


Die von den diversen religiösen Hoheiten vorgegebenen Anweisungen zur Lebensführung lassen sich nach Marti nicht mit allen Alltagspraktiken der Menschen zusammenbringen. Die Konfrontation mit neuen Umständen und Kontexten bringe das Individuum dazu, dass es eine reflexive Religiosität entwickle. Diese sei ein problemlösendes, dynamisches, differenziertes und unverzichtbares Element der persönlichen religiösen Entwicklung jedes Einzelnen. ${ }^{81}$

Die Betrachtung der empirischen Forschungsergebnisse unter dem Aspekt der reflexiven Religiosität zeigt, dass die Interviewpartnerinnen in ihren religiösen und kulturellen Angelegenheiten reflexiv handeln und sich dabei auf ihre intuitivindividuellen Entscheidungen verlassen. In keiner Plausibilisierung bzw. Modifikation religiöser Inhalte war zu erkennen, dass ein besonderer Bezug zu einer exklusiv orientierten religiösen Gemeinschaft hergestellt wurde. Die erlernten religiösen Inhalte bzw. religiösen Normen der Vergangenheit scheinen, wie es eben auch Gerardo Marti thematisiert, nicht mit der aktuellen Lebensführung übereinzustimmen. Die Interviewpartnerinnen bewegen sich räumlich nicht mehr nur in ihren ursprünglichen Bahnen, was zu verschiedensten Vertrauenspersonen in ihrem Leben führt - oder sie verlassen sich in ihren religiös motivierten Entscheidungen ausschließlich auf sich selbst. ${ }^{82}$

Insofern ist auch für die künftigen religiösen und kulturellen Entscheidungen der Interviewpartnerinnen eine Fortsetzung des reflexiven Handelns anzunehmen. Nur mittels dieser Vorgehensweise scheint es den Interviewpartnerinnen zu gelingen, ein emanzipatorisches Verhaltensmuster zu entwickeln, das gleichzeitig kein Loslassen der ursprünglich erlernten religiösen und kulturellen Praxis bedeuten muss. Zugleich stellt sich die Frage, ob die Modifikationshinweise längerfristig als Vorboten zur Entstehung hybrider religiöser Identitäten gedeutet werden können.

Die Vorgehensweise in der empirischen Analyse könnte ebenso auf Muslime unterschiedlicher Herkunft mit höherer Bildung ausgeweitet werden und aufschlussreiche Impulse mit sich bringen, inwiefern die religiösen Identitäten der muslimischen Akademiker sich generell im europäischen Kontext entfalten. Interessant wären außerdem empirische Untersuchungen zu Muslimen unterschiedlicher Herkunft mit niedrigeren Bildungsabschlüssen. Auch hier wäre zu analysieren, wie sich die Transformation von Religion im Migrationskontext gestaltet. Durch diese Herangehensweise könnte differenzierter analysiert werden, inwiefern Faktoren wie Armut, Wohlstand, Bildungsniveau über den Faktor Migration hinaus die Entstehung und Transformation religiöser Phänomene beeinflussen. Solche Untersuchungen wären ebenso relevant, weil sie eine Hilfestellung bzw. einen Anreiz zur Gestaltung religionspädagogischer und theologischer Diskurse im europäischen Kontext ermöglichen können.

81 Vgl. ebd., S. 6.

82 Vgl. Dursun 2015, S. 321. 


\section{Literaturverzeichnis}

Abū Dawūd: Sunan Abu Dawud. Volume II. Chapters: 519-1337. Ahmad Hasan, Ashraf, Muhammad (Hg.): Kitāb al Nikāh, Lahore 7, 1984. Chapter: 710/2143-2144.

Akyol, Taha: Modernleşme Sürecinde Türban. Istanbul 2008, S. 256-258.

Aslan, Ednan: „Muslime in Amerika und Österreich“, in: Rothgangel, Martin / Aslan, Ednan / Jäggle, Martin (Hg.): Religion und Gemeinschaft. Die Frage der Integration aus christlicher und muslimischer Perspektive (= Religion and Transformation in Contemporary European Society 3). Göttingen 2013, S. 51-67.

Beşer, Faruk: Hanımlara Özel Fetvalar. Istanbul ${ }^{41} 2013$.

Ceylan, Rauf: Islamische Religionspädagogik in Moscheen und Schulen. Ein sozialwissenschaftlicher Vergleich der Ausgangslage, Lehre und Ziele unter Berücksichtigung der Auswirkungen auf den Integrationsprozess der muslimischen Kinder und Jugendlichen in Deutschland (= Beiträge zur Islamischen Religionspädagogik 2). Hamburg 2008.

Çalışkan, Hasan: Aile ve Evlilik Hayatı. Ulm 2004.

Döndüren, Hamdi: Delilleriyle Aile Ilmihali-Nikah-Boşanma-Mahremiyetler-Kadın ve Çocuk Haklarl-Sorular-Cevaplar. Band I. Istanbul 1993.

Dursun, Sule: „Den eigenen Weg ... “ Deutungsmuster von Religion und Religiosität bei Frauen türkischer Herkunft mit Universitätsabschluss in Wien. DissertationsarbeitIslamische Religionspädagogik. Universität Wien 2015.

Froschauer, Ulrike / Lueger, Manfred: Das qualitative Interview. Wien 2003.

Güler, İlhami: Dine Yeni Yaklaşımlar. Eskişehir 2005.

Kalsky, Manuela: „Religiöse Flexibilität“, in: Reinhold, Berhardt / Perry-Schmidt, Leukel (Hg.): Multiple religiöse Identität, aus verschiedenen religiösen Traditionen schöpfen (= Beiträge zu einer Theologie der Religionen 5). Zürich 2003, S. 234-237.

Karakaşoğlu, Yasemin: „Islam und Moderne, Bildung und Integration. Einstellungen türkisch-muslimischer Studentinnen erziehungswissenschaftlicher Fächer", in: Rumpf, Mechthild / Gerhard, Ute / Jansen, Mechthild (Hg.): Facetten islamischer Welten-Geschlechterordnungen, Frauen- und Menschenrechte. Bielefeld 2003, S. 272289.

Kırbaşoğlu, Mehmet Hayri: „Ilmihal Dindarlığının Imkanı Üzerine“, in: Islamiyat, Üç Aylık Araştırma Dergisi. Band V/4. Ankara 2002, S. 109-122.

Mader, Katharina: Gender Budgeting: Ein emanzipatorisches, finanzpolitisches und demokratiepolitisches Instrument. Frankfurt am Main 2009.

Marti, Gerardo: „Religious Reflexivity: ,The Effect of Continual Novelty and Diversity on Individual Religiousity““, in: Sociology of Religion (76/1) 2015, S. 1-13.

Öztürk, Mustafa: „Egemen Bir Dini Söylem Tarzı Olarak Ataerkillik“, in: Islamiyat, Üç Aylık Araştırma Dergisi. Band IV/4. Ankara 2001, S. 111-117.

Subaşı, Necdet: „Mahalleyi Baskıyla Hatırlamak“, in: Takış, Tahsin (Hg.): Şerif Mardin Okumalarl. Ankara 2008.

Uysal, Asım / Uysal, Mürşide: Izahlı Kadın Ilmihali Ansiklopedisi. Konya 1993.

Yavuz, Fehmi: Din Eğitimi ve Toplumumuz. Ankara 1961. 


\section{Internetquellen}

Altıntaş, Ramazan: „Ilmihal Müslümanlığı Üzerine“, in: Memleket, 26. 05.2006, verfügbar unter: http://www.memleket.com.tr/ilmihal-muslumanligi-uzerine-2624yy.htm [10.01.2016].

Aslan, Ednan / Yıldız, Erol: Zwischenbericht für das Projektjahr 2013: Muslimische Alltagsspraxis in Österreich. Ein Kompass zur religiösen Diversität. Wien 2013, verfügbar unter: http://muslimische-milieus-in-oesterreich.univie.ac.at/fileadmin/user_upload/p_ iis/muslimische_alltagspraxis_in_oesterreich.projektbericht.pdf [18.01.2016].

Hölzl, Verena: „Burka-Verbot in Frankreich: Hinter dem Schleier“, in: Spiegel Online Panorama, 14.08.2015, verfügbar unter: http://www.spiegel.de/panorama/gesell schaft/burka-verbot-wie-wirkt-das-gesetz-in-frankreich-a-1009971.html [28.12.2015].

Jelen, Brigitte: „Educated, Independent, and Covered: the Professional Aspirations and Experiences of University-Educated Hijabi in Contemporary Turkey“ in: Women Studies International Forum (34/4) 2011, S. 308-319, verfügbar unter: http://www. sciencedirect.com/science/article/pii/S027753951100077X?np=y [21.09.2016].

Kyriadikidou, Athina: „Multiple discrimination against Muslim women in Europe: for equal opportunities“, Doc.12956, Parliamentary Assembly, Council of Europe; Committee on Equality and Non-Discrimination, 11.06.2012, available at: http:// semantic-pace.net/tools/pdf.aspx ?doc=aHR0cDovL2Fzc2VtYmx5LmNvZS5pbnQv bncveG1sL1hSZWYvWDJILURXLWV4dHIuYXNwP2ZpbGVpZD0xODc0OCZ sYW5nPUVO\&xsl=aHR0cDovL3NlbWFudGljcGFjZS5uZXQvWHNsdC9QZGY vWFJIZi1XRC1BVC1YTUwyUERGLnhzbA==\&xsltparams $=$ ZmlsZWlkPTE4N zQ4 [02.01.2016].

Marik-Lebeck, Stephan: „Die muslimische Bevölkerung Österreichs: Bestand und Veränderung 2001-2009“, in: Janda, Alexander / Vogl, Mathias (Hg.): Islam in Österreich, Österreichischer Integrationsfonds, 2010, verfügbar unter: file ://C:/Users/ User/Downloads/Islam_in_OEsterreich\%20(3).pdf [18.01.2016].

News: Burkaverbot abgewiesen-FPÖ hat für Antrag keine Mehrheit gefunden. Nur Unterstützung von Team Stronach, 10.07.2014, verfügbar unter: http://www.news.at/ a/nationalrat-burkaverbot-oesterreich-antrag-abgelehnt [28.12.2015].

OE24: Deutschland will Burka-Verbot einführen, 23.11.2015, verfügbar unter: http://www. oe24.at/welt/Deutschland-will-Burka-Verbot-einfuehren/213245279 [28.12.2015].

Öztürk, Mustafa: „Türkiye'de Dindarlık Tipolojileri“, in: HABERCI 28, 06.04.2012, verfügbar unter: http://haberci28.com/tr/yazigor.aspx ?yazid=457 [10.01.2016].

Topçuoğlu, Abdullah (Hg.): Türkiye'de Aile Değerleri Araştırması (Family Values in Turkey). T.C. Başbakanlık Aile ve Sosyal Araştırmalar Genel Müdürlüğü (T.R. Prime Ministry Directorate General of Family and Social Research), Ankara 2010, verfügbar unter: http://ailetoplum.aile.gov.tr/data/54293ea2369dc32358ee2b25/kutuphane_61_ turkiyede_aile_degerleri.pdf [10.01.2016].

Yeni, Şafak: Delilleriyle Aile Ilmihali, Promosyon Gazetesi, 2011, verfügbar unter: http://www.promosyongazetesi.com/yeni-safak-delilleriyle-aile-ilmihali/ [15.01.2016].

Zeynelabidin, Emel: „Im Iran oder Saudi-Arabien wäre ich längst tot“, in: Die Welt, 23.04.2015, verfügbar unter: http://www.welt.de/debatte/kommentare/article1399848 19/Im-Iran-oder-Saudi-Arabien-waere-ich-laengst-tot.html [28.12.2015]. 